\title{
SIMULATIONS OF NEUTRALIZED FINAL FOCUS
}

\author{
*D. R. Welch, D. V. Rose, T. C. Genoni, \\ Mission Research Corporation, \\ 5001 Indian School Rd. NE, Albuquerque, NM 87110 \\ S. S. Yu, and J. J. Barnard, \\ Lawrence Berkeley National Laboratory, \\ 1 Cyclotron Rd., Berkeley, CA 94720
}

In order to drive an inertial fusion target or study high energy density physics with heavy ion beams, the beam radius must be focused to $<3 \mathrm{~mm}$ and the pulselength must be compressed to $<10 \mathrm{~ns}$. The conventional scheme for temporal pulse compression makes use of an increasing ion velocity to compress the beam as it drifts and beam space charge to stagnate the compression before final focus. Beam compression in a neutralizing plasma does not require stagnation of the compression, enabling a more robust method. The final pulse shape at the target can be programmed by an applied velocity tilt. In this paper, neutralized drift compression is investigated. The sensitivity of the compression and focusing to beam momentum spread, plasma, and magnetic field conditions is studied with realistic driver examples. Using the 3D particle-in-cell code, we examine issues associated with self-field generation, stability, and vacuum-neutralized transport transition and focusing.

PACS numbers: $52.35 . Q z, 52.40 . \mathrm{Mj}, 52.59 . \mathrm{Sa}, 52.65 . \mathrm{Ww}$

*URL: www.mrcabq.com; Voice Mail: (505) 768-7723; Fax: (505)768-7601 


\section{INTRODUCTION}

Heavy ion fusion (HIF) requires the acceleration, transport, and focusing of many individual ion beams. Drift compression and beam combining prior to focusing results in 10--100 individual ion beams with $10^{-5}--10^{-4}-\mathrm{C} / \mathrm{m}$ line-charge densities [1]. A focusing force is applied to the individual ion beams outside of the chamber. These beams must propagate through the chamber and strike the target with $<5$-mm radius. The conventional scheme for temporal pulse compression makes use of an increasing ion velocity to compress the beam a factor of 10--20 as it drifts in vacuum and beam space charge to stagnate the compression [2]. Ideally, this situation leads to a mono-energetic beam for adequate transverse focusing.

In this paper, we consider neutralized drift compression (NDC) of an ion beam. Here, pulse compression and focusing is achieved in the presence of a neutralizing plasma over roughly 50--100 m just upstream of a preformed discharge channel specifically for pinched transport modes that guide the ion beam at small radius to the target [3]. Given adequate neutralization of the beam charge and current, the compression ratio is limited only by the accuracy of the applied velocity tilt and longitudinal temperature of the beam. The individual ion beams drift, compress, and combine before being captured in an adiabatic discharge lens still outside of the chamber and are injected at small radius into the chamber. Recent work [3] for assisted-pinched transport (APT) has shown that a discharge channel can efficiently capture and transport a very high current ion beam to a 5-mm spot on the hybrid fusion target [4]. In the APT scenario, possible deleterious effects of beam overlap and combining just before capture may include instabilities and increased emittance growth. Implicit particle-in-cell (PIC) simulations indicate that there 
are perveance limits above which beam filamentation can prevent good coupling to the discharge channel [5]. Transport in a solenoidal field, as in the modular solenoid accelerator scheme [6], is shown to greatly reduce this limit. The goal of these calculations is to elucidate the basic physics issues of plasma-neutralized beam combining within the context of a modular solenoid accelerator. The calculations are performed with the 3D parallel LSP [7] PIC code using a fully kinetic energy-conserving algorithm.

In Section II, we discuss the key physics issues regarding neutralization and beamplasma stability. The transition from vacuum to neutralized transport and transverse focusing of the longitudinally compressing beam are examined in Sec. III. Conclusions are presented in Sec. IV.

\section{PHYSICS ISSUES FOR NEUTRALIZED DRIFT COMPRESSION}

In this section, we examine the physics of a beam that has already acquired a head-totail velocity tilt for longitudinal time-of-flight compression. The time-dependent velocity function at a particular plane that produces a perfect beam longitudinal compression at a downstream distance $L$ is given by,

$$
v(t)=\frac{v(0)}{1-\frac{v(0) t}{L}},
$$

where $v(0)$ is the velocity of the pulse at $t=0$. In practice, the beam ion velocities will vary somewhat with some characteristic thermal velocity or error $\Delta v$ that limits the pulselength achievable to $t_{\min }=L \Delta v /\langle v\rangle$, where $\langle v\rangle$ is the mean beam velocity. 
Realistically, the applied voltage to an ion beam can be controlled with current accelerator technology to roughly $0.1 \%$.

The critical new physics involves the neutralization of the beam space charge by a plasma over many tens of meters. From a simple 1D analysis, if stipulated that the beam impulse due to space charge (resulting in electric field $E$ ) over the neutralized drift, $E L /\langle v\rangle$, must be less than the applied tilt, $\Delta v$, the neutralization fraction must be

$$
f>1-\frac{a^{2}}{2 L^{2} Z K(0)},
$$

where $K(0)$ is the initial beam perveance defined as $K=2 I_{b} / I_{A} \beta^{2}$ with Alfven current $I_{A}=$ $\beta \gamma m_{i} c^{3} / e Z$. Here, $I_{b}, a, \beta c, \gamma, m_{i}$ and $Z$ are the beam current, radius, velocity, relativistic factor, mass, and charge state, respectively. For typical HIF parameters, e.g., 780-A, 230$\mathrm{MeV}, 20-\mathrm{cm}$ radius $\mathrm{Ne}^{+}$beam with $L=55 \mathrm{~m}$, then $f>0.99$. The presence of a high density plasma $\left(n_{p}>>n_{b}\right)$ has been shown to provide this excellent charge neutralization in PIC simulations [8] of HIF beam transport. The plasma density condition requires increasingly higher plasma density as the beam compresses.

A second obstacle to beam compression is caused by the relative velocity of the beam ions and background plasma electrons. The electrostatic beam-plasma electron twostream or Buneman instability has a 1D dispersion relation given by

$$
\frac{\omega_{b}^{2}}{\left(\omega-k v_{b}\right)^{2}}+\frac{\omega_{p}^{2}}{\omega^{2}}=1,
$$

where $\omega_{b}$ is the beam-plasma frequency. The key dimensionless parameters to evaluate the instability growth are then for transport distance $\zeta=z \omega_{b} / v_{b}$ and for beam pulse duration $\tau=t \omega_{p}$. The instability can result in modulation of the beam momentum that 
ultimately limits the final pulse time. Changing beam and plasma frequencies, as well as 2D effects, have a stabilizing influence [9].

To evaluate both the gross charge neutralization and impact of the two-stream instability, we perform 1D simulations of the $\mathrm{Ne}^{+}$beam example with 110-ns initial pulse duration. The beam energy ramps up from 208 to $254 \mathrm{MeV}$--- roughly a $10 \%$ velocity tilt. A $0.1 \%$ random longitudinal velocity variation is given the beam that limits the compression to 50 (equivalent to $40 \mathrm{kA}$ peak current). The background plasma $\left(\mathrm{C}^{+}\right.$ plasma ions) is initialized with $3 \mathrm{eV}$-temperature and uniform densities of $6 \times 10^{8}$, $2.5 \times 10^{9}, 10^{10}$, and $4 \times 10^{10} \mathrm{~cm}^{-3}$. The beam has an initial $10^{9}-\mathrm{cm}^{-3}$ density. These cases are simulated with the LSP code utilized in 1D Cartesian coordinates with spatial and temporal resolutions adjusted to resolve both $\zeta$ and $\tau$.

For initial plasma densities up to $10^{10} \mathrm{~cm}^{-3}$, the maximum beam compression increases with the plasma density. The gross neutralization improves with density as seen in Fig. 1 with the neutralization fraction exceeding $99 \%$ for $>10^{10}-\mathrm{cm}^{-3}$ plasma density. For the lower density cases, the neutralization was inadequate at $90 \%$ and $80 \%$ for $2.5 \times 10^{9}$ $\mathrm{cm}^{-3}$ and $6 \times 10^{8}-\mathrm{cm}^{-3}$ densities, respectively. Figure 1 also shows the beam compression was reduced from a ratio of 48 (50 is the maximum possible compression) for the higher density simulations to 23 and 8 for the lower densities. For the higher density cases, a nearly 40-kA peak current was achieved with increasing longitudinal "wings" at both beam ends.

For the highest density case, the effect of the two-stream interaction is seen from in the beam phase space when compared with a simulation without self electromagnetic fields (ballistic case). Although the full-width-half-maxima of the phase space and 
current pulse with the instability present are similar to that calculated for the ballistic simulation, the saturated state of the instability results in a fraction of the beam ions being driven from the local mean velocity. These high longitudinal emittance ions are the cause of the "wings" in Fig. 1 for the higher density plasma simulations. Higher dimensional effects, varying plasma density, variable magnetic topology, and beam radius could reduce the impact of the two-stream further. These effects are the subject of future research.

Recent simulations [5] exhibited strong magnetic filamentation particularly with higher perveance beams. Because the filaments have characteristic scale length given by the collisionless plasma skin depth, the instability is likely Weibel [10] in nature. The impact of self fields severely impacts the drift length and beam perveance that can be efficiently focused. Adding a solenoidal magnetic field has a dramatic effect on reducing the self-field growth by constraining plasma electrons to move axially. Analytic theory of equilibrium fields of Kagonovich [11] shows that the exposed axial net current is greatly reduced for $\omega_{p} \beta / \omega_{c}<1$, i.e., when the cyclotron radius is less than a plasma skin depth. This effect is calculated in a series of 2D LSP simulations for a high current (already compressed) HIF driver-scale 150-kA, 250-MeV, 10-cm outer radius $\mathrm{Ne}^{+}$beam $\left(n_{b}=7 \times 10^{11} \mathrm{~cm}^{-3}\right)$ injected into a $15-\mathrm{cm}$ outer radius pipe filled with a $10^{12}-\mathrm{cm}^{-3}$ density plasma. An applied solenoidal field of 0,2 , and $8 \mathrm{kG}$ was used which yielded a $\omega_{p} \beta / \omega_{c}=$ $\infty, 0.24$, and 0.05 . The net current with no applied field reaches roughly $7 \mathrm{kA}$, flowing in a thin sheath at the beam edge. As the solenoidal field increases, the sheath current drops to small values as predicted. The physical reason for the drop in net current is that, as $B_{z}$ increases, electrons become constrained to move only in the axial direction forcing some 
electrons to move at the beam velocity to keep up with the beam and maintain charge neutralization. Without a solenoidal field, the charge neutralization, nearly perfect, was mainly from the radial direction. In the strong applied field case with $\mathrm{n}_{\mathrm{p}} \approx \mathrm{n}_{\mathrm{b}}$, we are left with the minimum residual potential (or trapping potential) $1 / 2 m_{e} v_{b}^{2}$ for an ion beam that is provided a purely axial source of electrons for neutralization [12]. For increasing plasma density, a smaller fraction of the plasma electrons are required to co-move with the beam. For this reason, further LSP simulations confirm that to maintain excellent charge neutralization, a second constraint of $n_{p} / n_{b}>>1$ is required as suggested by the earlier 1D simulations.

\section{TRANSITION FROM VACUUM BRILLOUIN FLOW TO NEUTRALIZED TRANSPORT AND FOCUSING}

Recent HIF accelerator studies have begun that make use of solenoidal transport with ions in Brillouin flow (BF) equilibrium. This equilibrium for ion beams up to a few 100 $\mathrm{MeV}$ provides transport for considerably higher line-charge densities $(\lambda)$ than the standard alternating gradient quadrapole approach [6]. The appropriate beam envelope equation is [13]

$a^{\prime \prime}+\frac{\Omega_{L}^{2}}{\beta^{2} c^{2}} a-\frac{K}{a}-\frac{\varepsilon^{2}}{\beta^{2} \gamma^{2} a^{3}}=0$,

where $\Omega_{L}=e B_{z} / 2 m_{i}$, and $\varepsilon$ are the beam Larmor frequency, and normalized emittance, respectively. For a cold unneutralized beam in equilibrium, the required magnetic field is given by $B_{z}=2 \beta c m_{i} K^{1 / 2} / e a$. To accommodate an velocity tilt on the beam, the perveance must vary as $K=\Omega_{L}^{2} a^{2} / \beta^{2} c^{2}$, which requires the beam current to rise with beam $\gamma \beta$, i.e., 
keeping $\lambda$ constant. As the beam $K$ becomes neutralized in the presence of a plasma, the beam feels a strong net focusing force unless the solenoidal field is greatly reduced. The decreasing magnetic fields have a deleterious effect on backstreaming plasma electrons that compresses them to small radius within the beam. This electron behavior increases the beam emittance. A plasma transition in this case requires nearly absolute suppression of backstreaming electrons. We accomplish this by placing just upstream of the plasma a dipole magnetic field that has been shown to be effective in previous simulations [14].

We now quantify the magnitude of the dipole magnetic field required to suppress upstream plasma electron motion. In a 3-m PIC plasma simulation, the solenoidal field on axis transitions from $2.5 \mathrm{~T}$ at $\mathrm{z}<1 \mathrm{~m}$ to $0.125 \mathrm{~T}$ by $\mathrm{z}=250 \mathrm{~cm}$ in the $\mathrm{NDC}$ region. The axial variation of the magnetic dipole field (normalized to a maximum of 1) is shown in Fig. 2 that produces a minimal net impulse and offset to the beam. As in an earlier example, the $\mathrm{Ne}^{+}$beam has 780 -A current, 20 -cm sharp outer radius, $8-\pi$-mm-mrad emittance, $230-\mathrm{MeV}$ mean energy, 110 -ns pulsewidth, with a $10 \%$ perfect velocity tilt ( $L$ $=55 \mathrm{~m}$ ). The beam has $18 \mathrm{~kJ}$ total energy, somewhat small for a driver, but sufficiently high to elucidate the physics. The plasma is initialized for $\mathrm{z}>240 \mathrm{~cm}$ with $10^{10}-\mathrm{cm}^{-3}$ number density with infinite mass ions but kinetic electrons.

The peak dipole magnetic field was increased from 1.2--4.8 kG in three simulations. As the field increased, the number of backstreaming electrons dropped to 0 and the beam quality improved. For a 1.2-kG dipole, we see in Fig. 3 that a significant density of backstreaming electrons was accelerated by the beam space charge from the plasma surface and compressed in the solenoidal field. The impact of the electrons' self fields 
was visible in the beam density plots. The electrons are completely suppressed in the 4.8-kG dipole simulation with no significant emittance growth calculated.

A final issue concerning NDC involves the final transverse focusing of the beam just before striking an HIF target or entering an adiabatic section of the discharge channel for further focusing. This focusing must accommodate a large energy spread (10--20\%) for the 50--100-m drift length and hit a 1-cm radius at the discharge channel entrance. From the immersed envelope equation (Eq. 4), we quickly solve for the energy acceptance of the neutralized solenoidal focusing assuming small energy variation $\Delta \mathrm{E}$ about $\mathrm{E}$. With small beam emittance, $\mathrm{K}=0$, and constant $\Omega_{\mathrm{L}}$, the entire beam is captured within some final radius given

$\frac{\Delta E}{E}<\frac{8}{\pi r}$

with $r$ being the ratio of the initial-to-final radius. Practically, the beam emittance ultimately limits the maximum tolerable focal length.

For HIF, the above criterion sets a limit on the maximum energy variation of a beam coupling to a fusion target directly or with an intermediate step to discharge channel. In the case of multiple beams combining in a single solenoid, the initial radius is actually the envelope of all the combining beams. For the hybrid target [4], requiring a $0.5-\mathrm{cm}$ radius spot at the target and assuming a $10-\mathrm{cm}$ radius beam, the accepted energy variation is roughly $13 \%$. Coupling into a discharge channel of $1-\mathrm{cm}$ radius increases the accepted spread to $26 \%$. The discharge channel then compresses the beam to the $0.5-\mathrm{cm}$ radius. Thus, the discharge channel also relaxes the required beam emittance. 
We now check the envelope equation results with an LSP simulation of a plasmaneutralized solenoidal final focus. $\mathrm{A} \mathrm{Ne}^{+}$beam with $230-\mathrm{MeV}$ mean energy is injected in $\mathrm{BF}$ equilibrium with a uniform 5-T solenoidal field through a zero potential plane, directly into a plasma drift section. This situation removes the need to model the transition region from vacuum to NDC. The beam has a $10-\mathrm{cm}$ radius, $14-\pi-\mathrm{mm}-\mathrm{mrad}$ emittance, 780-A initial current, 20-ns pulse (short for computational speed) with a 1-ns rise/fall current. The beam is given a $20 \%$ head-to-tail energy tilt in this case and $L=10$ m. Eq. 5 predicts the entire beam should strike with $8 \mathrm{~mm}$ radius. The plasma has a density rising linearly from $3 \times 10^{11}$ to $3 \times 10^{12} \mathrm{~cm}^{-3}$ from $z=0--600 \mathrm{~cm}$ then held constant through the transverse focus at $\mathrm{z}=615 \mathrm{~cm}$. Due to the plasma neutralization, the beam feels a net $\mathbf{v} \times \mathbf{B}$ focusing force. With self fields turned off, the beam edge radius ( $\left.a=2^{1 / 2} r_{r m s}\right)$ comes to a minimum of $4 \mathrm{~mm}$ at mean energy expanding to $8 \mathrm{~mm}$ at the pulse extremes, again consistent with the above equation. With self fields turned on, the beam focus is pushed forward to $\mathrm{z}=620 \mathrm{~cm}$. As seen in Fig. 4 by $160 \mathrm{~ns}$, the beam has compressed both axially to 5 -ns pulselength and radially to $<1.0 \mathrm{~cm}$. At the $z=620-\mathrm{cm}$ plane, the rms radius is $<7 \mathrm{~mm}$ with a slight emittance gain in the tail to $20 \pi$-mm-mrad and no damage to the longitudinal phase space. We find $99 \%$ of the beam is focused within the required $1-\mathrm{cm}$ radius. The beam emittance growth is the result of too little plasma density near focus where $n_{b}>n_{p}$. A larger plasma density would reduce the effect.

\section{CONCLUSIONS}

We have studied the basic physics associated with neutralized drift compression of ion beams. Key transport areas included the transition of heavy ion beams from BF to 
NDC, beam-plasma instability growth and final focus in a modular solenoid accelerator configuration. Transport of ion beams in a solenoidal field removes most of the self magnetic fields that lead to filamentation but requires the plasma density to be 10 times that of the beam to maintain good electrostatic neutralization. For reasonable ratios of plasma-to-beam density, the saturated state of the two-stream instability does not significantly degrade the beam phase space in 1D simulations. Simulations of the transition show the necessity of including a $>2-\mathrm{kG}$ dipole magnetic field to prevent electron acceleration upstream into the beam. If unchecked, the solenoidal field transition compresses these electrons producing anharmonic potentials that degrade the beam. Confirming envelope calculations, LSP simulations show that a strong solenoidal field can effectively focus (transversely) an ion beam with a $20 \%$ energy tilt in the presence of a plasma as it continues to longitudinally drift compress.

Work has begun on integrated simulation of the BF, NDC, final focus, and chamber transport of HIF beams. Recently, such simulations, not discussed here, have shown good overall coupling to the hybrid target. Utilizing a conductivity model to approximate the response of the plasma, the simulations predict $>90 \%$ transport efficiency to within a 5-mm spot for realistic HIF driver parameters. Near term, a proposed upgrade of the NTX experiment with 0.5 to $1 \mathrm{MeV} \mathrm{He}^{+}$ions is currently being designed with beam parameters that should enable sufficient target heating to access the HEDP (high energy density physics) regime with compressed beam pulses of 1-ns duration and 1-mm radius given $0.1 \%$ velocity control. Overall, this work suggests that NDC of heavy ion beams is a promising research path for both HIF and HEDP. 


\section{ACKNOWLEDGEMENTS}

We express our appreciation for useful discussions and support to Grant Logan, Ed Lee, Alex Friedman, Enrique Henestroza, Christine Celata, and Igor Kagonovich. Work supported by the Virtual National Laboratory for HIF, the Department of Energy through Princeton Plasma Physics Laboratory, and Lawrence Berkeley National Laboratory.

\section{REFERENCES}

[1] S. S. Yu, W. R. Meirer, R. P. Abbott, J. J. Barnard, T. Brown, D. A. Callahan, C. Debonnel, P. Heitzenroeder, J. F. Latowski, B. G. Logan, S. J. Pemberton, P. F. Peterson, D. V. Rose, G.-L. Sabbi, W. M. Sharp, D. R. Welch, Fusion Sci. and Techno. 44, 266 (2003).

[2] See C. M. Celeta, F. M. Bieniosek, E. Henestroza, J. Kwan, E. P. Lee, G. Logan, L.

Prost, P. A. Seidl, J.-L. Vay, W. L. Waldron, S. S. Yu, J. J. Barnard, R. Cohen, A. Friedman, D. P. Grote, S. M. Lund, A. Molvick, W. Sharp, G. Westenskow, R. C. Davidson, E. Gilson, P. Efthimion, R. Majeski, H. Qin, P. G. O'Shea, R. A. Kishek, M. Reiser, B. Beaudoin, S. Bernal, Y. Cui, A. Diep, D. Feldman, M. Glazner, T. F. Godlove, I. Haber, J. Harris, H. Li, J. Neumann, B. Quinn, M. Qurius, M. Snowel, A. Valfells, M. Virgo, M. Walter, R. Yun, Y. Zou, D. V. Rose, and D. R. Welch, Phys. Plasmas 10, 2064 (2003).

[3] D. R. Welch, T. C. Genoni, D. V. Rose, B. V. Oliver, R. E. Clark, C. L. Olson, and S. S. Yu, Phys. Plasmas 10, 2442 (2003). 
[4] D.A. Callahan, M.C. Herrmann, and M. Tabak, Laser and Particle Beams 20, 405-410 (2002).

[5] D. R. Welch, D. V. Rose, S. S. Yu, and C. L. Olson, "Multiple beam interaction studies in heavy ion fusion," in the Proceedings of the 2003 Particle Accelerator Conference, Portland, OR, May 2003.

[6] B. G. Logan, private communication, (2003). E. P. Lee and R. Briggs, "The solenoidal transport option: IFE drivers, near-term research facilities, and beam dynamics," Lawrence Berkeley National Laboratory Report LBNL-40774, HIFAN 914, Sept. 1997.

[7] D. R. Welch, D. V. Rose, B. V. Oliver, and R. E. Clark, Nucl. Instrum. Meth. Phys. Res. A 464, 134 (2001); LSP is a software product of Mission Research Corporation (http: \www.mrcabq.com).

[8] D. R. Welch, D. V. Rose, W. M. Sharp, C. L. Olson, and S. S. Yu, Laser and Particle Beams, 20, 621 (2002).

[9] P. Stroud, Laser and Particle Beams 4, 261 (1986).

[10] E. S. Weibel, Phys. Fluids 10, 741 (1967).

[11] I. Kaganovich, private communication (2003); these proceedings (2004).

[12] C. L. Olson, Heavy Ion Inertial Fusion, AIP Conference Proceedings 152, p. 215 (American Institute of Physics, New York, 1986).

[13] M. Reiser, Theory and Design of Charged Particle Beams, (John Wiley and Sons, Inc., New York, 1994).

[14] D. V. Rose, D. R. Welch, C. L. Olson, S. S. Yu, S. Neff, W. M. Sharp, and the ARIES Team, "Impact of beam transport method on chamber and driver design for heavy ion inertial fusion energy," to appear in Fusion Science and Technology (2004). 

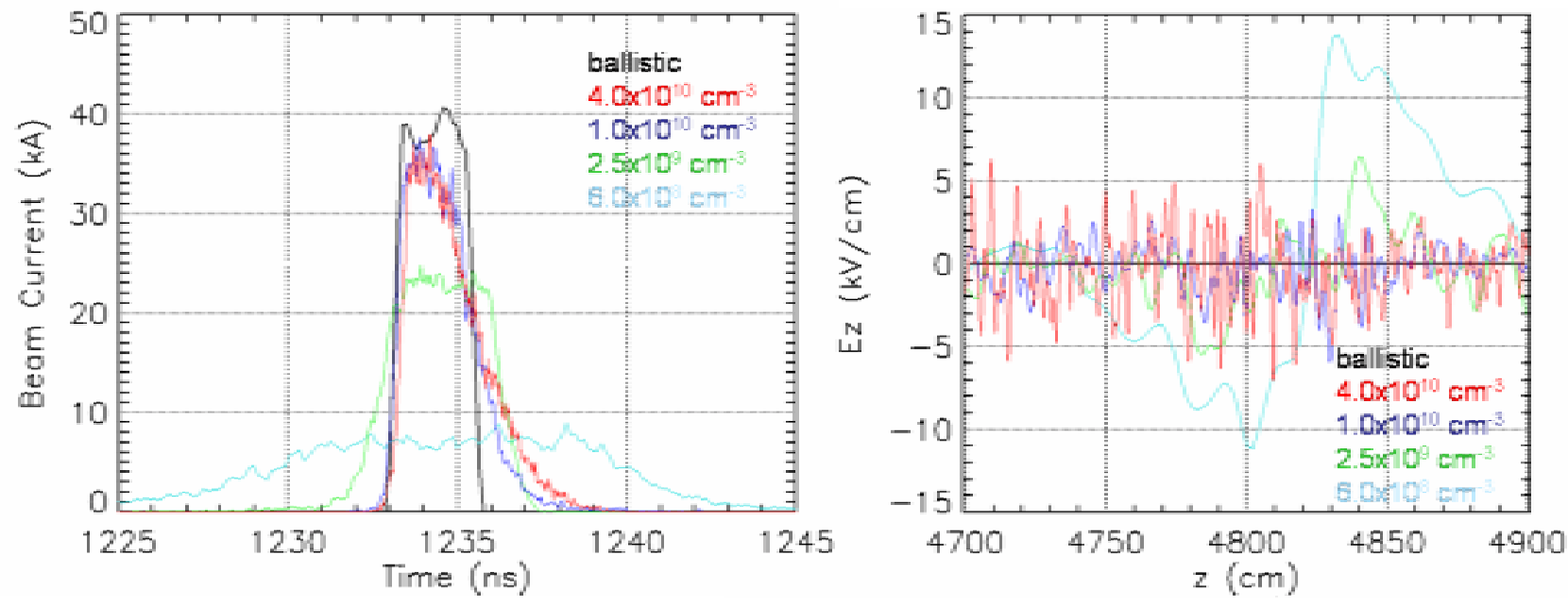

Figure 1. For the 1D LSP simulations of two-stream, the beam current at focus length $L$ is plotted on the left, and the longitudinal electric fields at time $1100 \mathrm{~ns}$ is shown on the right. The simulations were for ballistic transport and with self fields at the indicated plasma densities. At injection, the beam was given a random velocity variation of $0.1 \%$. 


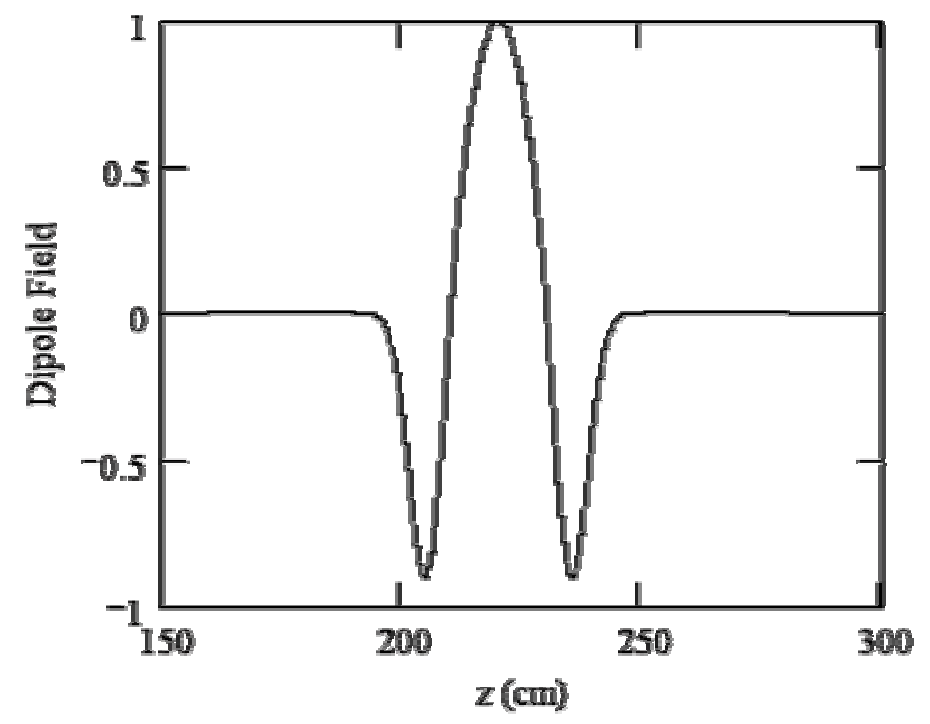

Figure 2. The on-axis solenoidal field for the transition simulation is shown. The dipole field axial dependence normalized to unity minimizes beam impulse and offset while preventing plasma electron back streaming. 


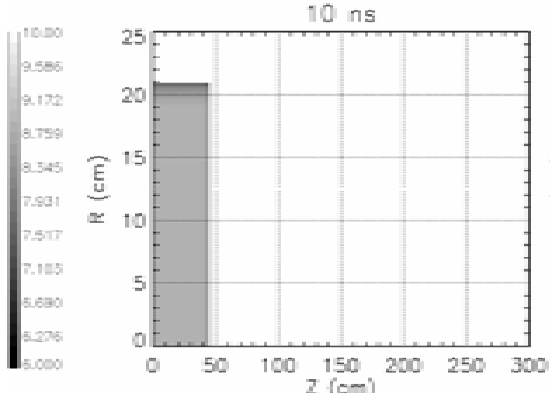

(a)

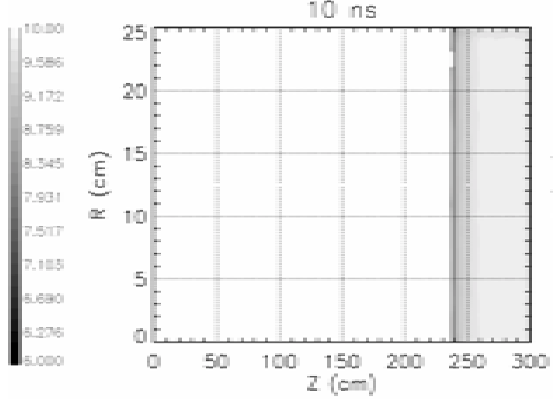

(d)

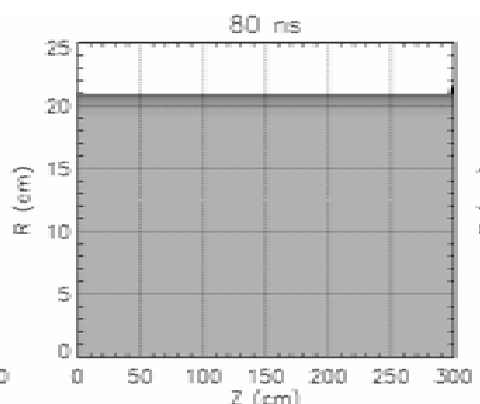

(b)

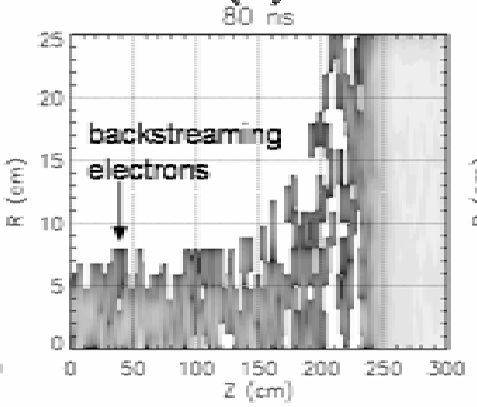

(e)

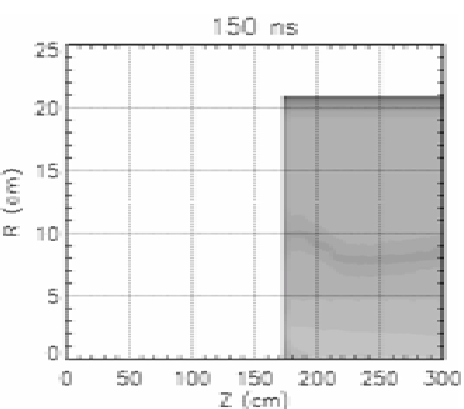

(c)

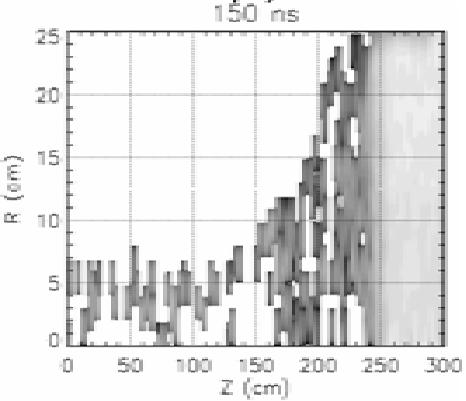

(f)

Figure 3. The logs of the beam density (a-c) and plasma electron density (d-e) are plotted for (left to right) 10,80 , and $150 \mathrm{~ns}$. This $3 \mathrm{D}$ transition simulation had a $1.2-\mathrm{kG}$ dipole field. 


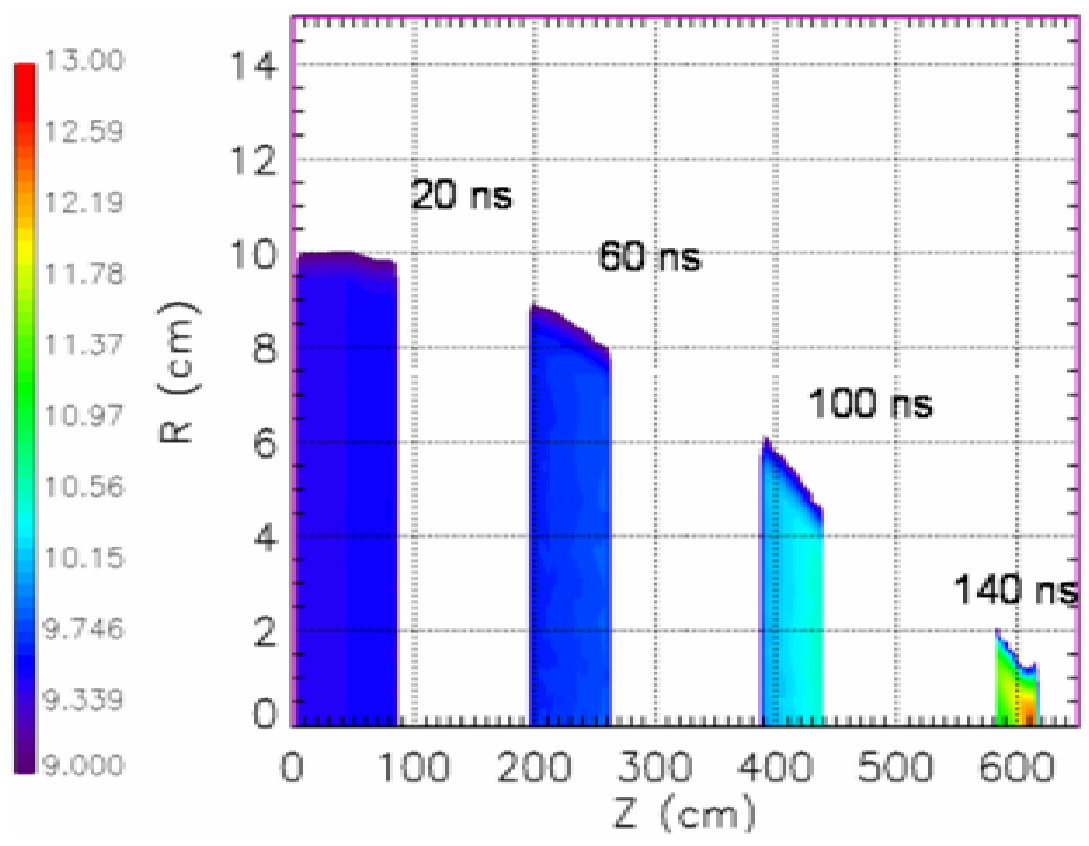

Figure 4. In the PIC plasma simulation in a uniform 5-T solenoidal field, the log of the beam density $\left(\mathrm{cm}^{-3}\right)$ is shown after 20,60, 100, and 140ns. The initial plasma density increases from $3 \times 10^{11} \mathrm{~cm}^{-3}$ at $\mathrm{z}=0 \mathrm{~cm}$ to $3 \times 10^{12} \mathrm{~cm}^{-3}$ at $\mathrm{z}=600 \mathrm{~cm}$. The $780 \mathrm{~A} \mathrm{Ne}^{+}$ beam has an initial $10-\mathrm{cm}$ edge radius, $230-\mathrm{MeV}$ mean energy with a $20 \%$ energy tilt. 